\title{
Effects of bone Young's modulus on finite element analysis in the lateral ankle biomechanics
}

\author{
W.X. Niu ${ }^{\mathrm{a}, \mathrm{b}, \mathrm{c}}$, L.J. Wang ${ }^{\mathrm{d}}$, T.N. Feng ${ }^{\mathrm{a}, \mathrm{c}}$, C.H. Jiang ${ }^{\mathrm{a}}$, Y.B. Fan ${ }^{\mathrm{e}, *}$ and M. Zhang ${ }^{\mathrm{b}, *}$ \\ ${ }^{\mathrm{a}}$ Tongji Hospital, Tongji University School of Medicine, Shanghai, China \\ ${ }^{\mathrm{b}}$ Interdisciplinary Division of Biomedical Engineering, The Hong Kong Polytechnic University, Hong Kong, China \\ ${ }^{\mathrm{c}}$ Shanghai Key Laboratory of Orthopaedic Implants, Shanghai, China \\ ${ }^{\mathrm{d}}$ Physical Education Department, Tongji University, Shanghai, China \\ ${ }^{\mathrm{e}}$ Key Laboratory for Biomechanics and Mechanobiology of Ministry of Education, School of Biological Science \\ and Medical Engineering, Beihang University, Beijing, China
}

\begin{abstract}
Finite element analysis (FEA) is a powerful tool in biomechanics. The mechanical properties of biological tissue used in FEA modeling are mainly from experimental data, which vary greatly and are sometimes uncertain. The purpose of this study was to research how Young's modulus affects the computations of a foot-ankle FEA model. A computer simulation and an in-vitro experiment were carried out to investigate the effects of incremental Young's modulus of bone on the stress and strain outcomes in the computational simulation. A precise 3-dimensional finite element model was constructed based on an in-vitro specimen of human foot and ankle. Young's moduli were assigned as four levels of 7.3, 14.6, 21.9 and 29.2 GPa respectively. The proximal tibia and fibula were completely limited to six degrees of freedom, and the ankle was loaded to inversion $10^{\circ}$ and $20^{\circ}$ through the calcaneus. Six cadaveric foot-ankle specimens were loaded as same as the finite element model, and strain was measured at two positions of the distal fibula. The bone stress was less affected by assignment of Young's modulus. With increasing of Young's modulus, the bone strain decreased linearly. Young's modulus of $29.2 \mathrm{GPa}$ was advisable to get the satisfactory surface strain results. In the future study, more ideal model should be constructed to represent the nonlinearity, anisotropy and inhomogeneity, as the same time to provide reasonable outputs of the interested parameters.
\end{abstract}

Keywords: Foot and ankle, finite element analysis, Young's modulus, strain, ankle inversion

\section{Introduction}

Finite element analysis (FEA) has been widely used in biomechanics [1-8]. The biological tissue and

*Corresponding author: Y.B. Fan, Key Laboratory for Biomechanics and Mechanobiology of Ministry of Education, School of Biological Science and Medical Engineering, Beihang University, Beijing 100191, China. Tel./Fax: +86 108233 9428; E-mail: yubofan@buaa.edu.cn; M. Zhang, Interdisciplinary Division of Biomedical Engineering, The Hong Kong Polytechnic University, Hong Kong, China. Tel./Fax: +86 8522766 4939; E-mail: ming.zhang@polyu.edu.hk. organs all have very complicated shapes, structures and mechanical properties. These characters make the application of FEA in biomechanics very challenging [9-11]. In the FEA modeling, the mechanical properties of various biological tissues are mostly from experimental data in published documents. These data often vary greatly and are sometimes uncertain, because the measured property has considerable variability influenced by degeneration, gender, race, measurer, and experimental condition.

The mechanical property allocation has potential influences on the FEA outcomes [4]. In the foot-ankle 
biomechanics, bones are not normally distinguished to cortical and cancellous bones. They are usually assumed as being homogeneous, isotropous and linear elastic. Though Young's modulus of bone is always assigned with 7.3 GP [6, 7], the initiator could not provide any source of origin for this value [12]. In most documents, the Young's modulus of bone was measured from 6 to $27.6 \mathrm{GPa}$ [13]. The moduli from nanoindentation experiments on human tibia were even from 13.1 to $32.2 \mathrm{GPa}$ [14]. The default value of $7.3 \mathrm{GPa}$ is a bit lower for this interval.

This study aimed to research how Young's modulus affects the computations of a foot-ankle FEA model. A computer simulation and an in-vitro experiment were carried out to study the stress/strain of the caput fibulae while ankle inversion. This condition was analyzed here because ankle inversion commonly incurred injuries in the caput fibulae and it was a representative question in the foot-ankle biomechanics $[15,16]$.

\section{Materials and methods}

\subsection{FE modeling and analysis}

The geometry of the FE model was obtained from three-dimensional reconstruction of computer tomography (CT) images from the right foot of a female cadaver (age: 56 years, height: $164 \mathrm{~cm}$; and body mass: $58 \mathrm{~kg}$ ). The donor had no foot affliction, abnormalities, trauma, or systemic disease in the ankle or/and foot. The in-vitro foot-ankle subject showed an unforced supination posture with an ankle plantar-flexion of $27^{\circ}$ and a subtalar-joint inversion of $18^{\circ}$. A HiSpeed Dual Product scanner (General Electric, Germany) was used to scan the cross sections of specimen with intervals of $0.625 \mathrm{~mm}$.

All 700 images were segmented using MIMICS 10.01 (Materialise, Leuven, Belgium) to obtain the boundary point clouds for all bones. The point clouds were proceed using the inverse engineering soft- ware Geomagic Studio 11.0 (Raindrop Geomagic Inc., Research Triangle Park, NC, USA) to generate groups of detailed polygonal surfaces, and to subsequently smooth the noisy surface data. Distal tibia and fibula with marrow cavities were constructed with Boolean operation. Another inverse engineering software Rapidform XOR2 (INUS Technology, Seoul, Korea) was applied to produce 71 surface models of ligaments and plantar fascia according to various anatomical positions and directions.

All geometric models of bones, ligaments and fascias were imported to the FE software ABAQUS 6.11 (Simula, Providence, RI, USA), in which the FE model was finally constructed and computed. All bone blocks were meshed with tetrahedral elements. The Poisson's ratio for the bony structures was assigned as 0.3 , and the Young's moduli were assigned as 7.3, 14.6, 21.9 and 29.2 GPa progressively for four models (Table 1). The bone surfaces were selected to be meshed as tetragon elements and the elements were extruded to generate cartilage models of hexahedron elements. The similar process was operated to produce the hexahedron elements of ligaments and plantar fascia. The thickness of ligaments and plantar fascia referred to experimental measurements by other authors [17-19]. Geometric parameters of main ligaments in foot were reported by Mkandawire and Ledoux [18]. Geometric and viscoelastic parameters of main ligaments in the ankle joint were described in other documents $[18,19,25]$. The data were compared among different documents, and some ligaments thicknesses without reference were selected according to the data of neighboring or similar ligaments. The entire FE model was shown as Fig. 1.

The center of ankle joint (CAJ) for the model was calculated from the medial and lateral malleoli [26]. In terms of the boundary conditions, the superior surfaces of the tibia, the fibula and CAJ were fixed completely. A tie constraint was constructed between CAJ and the calcaneum bone. The calcaneum bone was loaded to rotate around CAJ for inversion of $10^{\circ}$ and $20^{\circ}$

Table 1

Mechanical and geometric parameters of tissues for finite element modeling

\begin{tabular}{|c|c|c|c|c|c|}
\hline Tissue & Element types & Young's modulus (MPa) & Poisson ratio & Thickness (mm) & $\overline{\text { References }}$ \\
\hline Bone & Tetrahedron & $7,300 / 14,600 / 21,900 / 29,200$ & 0.30 & - & {$[4,13]$} \\
\hline Ankle Articular Cartilage & Hexahedron & 1 & 0.03 & 1.2 & {$[20,21]$} \\
\hline Foot Articular Cartilage & Hexahedron & 1 & 0.08 & $0.4-1.0$ & {$[22,23]$} \\
\hline Plantar Fascia & Hexahedron & 350 & 0.49 & $2.02-2.57$ & {$[17,24]$} \\
\hline Foot Ligament & Hexahedron & 260 & 0.49 & Table 2 & {$[4,18]$} \\
\hline
\end{tabular}




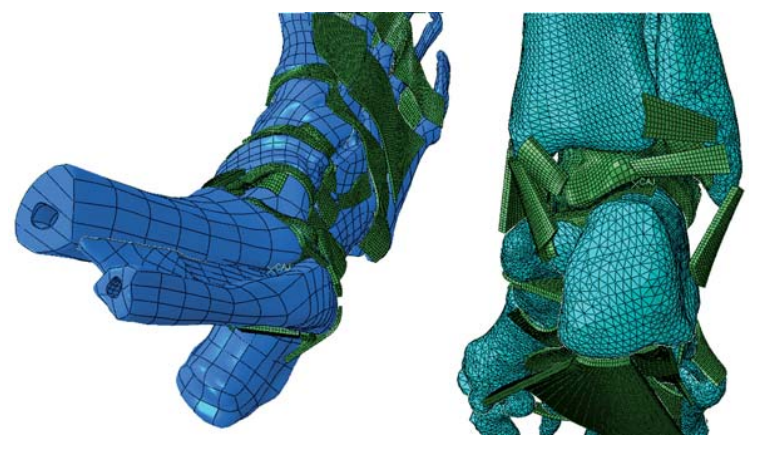

Fig. 1. The finite element model of foot and ankle.

respectively. The calculated outcomes included the principal strain, the principal stress and von Mises stress of the fibula, stress and strain of calcaneofibular ligament (CFL) and the contact stress of the tibiotalar and subtalar articular cartilages.

\subsection{In-vitro experiment}

Six fresh-frozen human cadaveric shank-foot samples (3 left laterals; and 3 right laterals) were measured. The donors included 2 men and 4 women, with a mean age of 50 years (range 39-67 years). Each specimen was radiographed to ensure that no specimens had bone defects such as previous fractures, deformities or tumors. After shipmen of frozen specimens to us, they were immediately placed at $-20^{\circ} \mathrm{C}$ until the specimen preparation.

For each specimen, the shank was cut transversely approximately $20 \mathrm{~cm}$ proximal to the ankle joint. The skin and subcutaneous tissue were dissected free from the lateral ankle to expose ligaments and fibula bone surface, and the joint capsule and ligaments of the thawed cadaver ankle were left intact. After the tissue has been removed, the region is degreased with alcohol and acetone. As shown in Fig. 2, two resistance strain gauges were bonded to the caput fibulae with cyanoacrylate in the direction along the extension CFL. The gauges were connected to static strain indicator (DH-3818, Donghuatest Corp., Jingjiang, Jiangsu, China) with $1 / 4$ bridge converter and common compensating gauge. A temperature compensating gauge was bonded to a fibula section cut from the same specimen.

Figure 2 also showed that the tibia and fibula residuals were fixed completely with a bench vice, a steel pipe and eight trip bolts. The lateral side of the sample was placed at the top. The sample dropped spontaneously

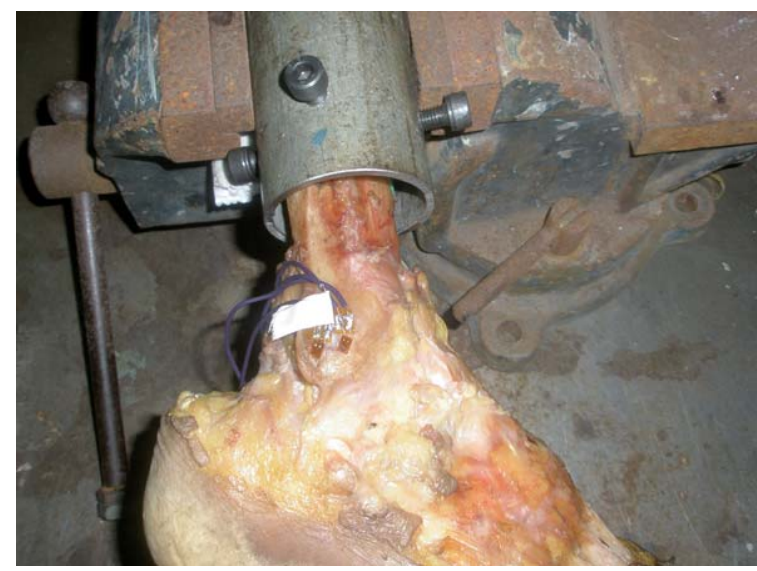

Fig. 2. Setup of an in-vitro specimen and fixation of strain gauges.

since the foot gravity, and presented a supination condition. A material test machine (CSS-44010, CRITM, Changchun, Jilin, China) was used to load on the calcaneum bone for inversion of $10^{\circ}$ and $20^{\circ}$ respectively. The loading rate was $2 \mathrm{~mm} / \mathrm{min}$. Before the formal trial, each specimen was preconditioned to inversion $10^{\circ}$ for five cycles with the same loading rate.

\section{Results}

\subsection{FEA results}

The FEA computations at four levels of Young's modulus were listed as Table 2. Changes of the peak 1st and the absolute $3 \mathrm{rd}$ principal strains of the fibula with increase of the bone Young's modulus were plotted as Fig. 3. When the Young's modulus was assigned as $29.2 \mathrm{GPa}$ and the ankle joint was inversed with $20^{\circ}$, the von Mises stress around the ankle region was shown as Fig. 4.

The stress and strain peaks of various tissues in the computational simulation were listed Table 2. With increasing of bone Young's modulus, the peak von Mises stress, 1st and absolute 3rd principal stress of the fibula were all increased The same tendencies were also found in the peak von Mises stress and 1st principal strain of CFL increased. However, the 1st principal strain and absolute 3rd principal strain decreased obviously with increasing of bone Young's modulus.

Figure 5 showed that the 1 st principal strain of the fibula had the peak at the caput fibulae. Both the position and direction agreed well between the calculation 
Table 2

Peak stress and strain of various tissues around ankle joint in finite element analysis

\begin{tabular}{|c|c|c|c|c|c|c|c|c|c|}
\hline \multirow{2}{*}{\multicolumn{2}{|c|}{$\begin{array}{l}\text { Ankle inversion }\left(^{\circ}\right) \\
\text { Young's Modulus of Bone (GP }\end{array}$}} & \multicolumn{4}{|c|}{10} & \multicolumn{4}{|c|}{20} \\
\hline & & 7.3 & 14.6 & 21.9 & 29.2 & 7.3 & 14.6 & 21.9 & 29.2 \\
\hline \multicolumn{2}{|c|}{$\begin{array}{l}\text { Young's Modulus of Bone (GPa) } \\
\text { Von Mises stress of fibula (MPa) }\end{array}$} & 14.0 & 15.4 & 16.2 & 16.8 & 28.1 & 30.8 & 32.3 & 33.3 \\
\hline \multicolumn{2}{|c|}{ 1st principal stress of fibula (MPa) } & 16.3 & 17.6 & 18.5 & 19.2 & 32.8 & 35.5 & 37.0 & 38.0 \\
\hline \multicolumn{2}{|c|}{ 3rd Principal stress of fibula (MPa) } & -11.2 & -12.7 & -13.5 & -9.4 & -23.7 & -26.8 & -28.5 & -29.6 \\
\hline \multicolumn{2}{|c|}{ 1st principal strain of fibula $(\mu \varepsilon)$} & 2038 & 1109 & 773 & 596 & 4102 & 2221 & 1543 & 1188 \\
\hline \multicolumn{2}{|c|}{ 3rd principal strain of fibula $(\mu \varepsilon)$} & -1595 & -873 & -609 & -470 & -3293 & -1807 & -1260 & -972 \\
\hline \multicolumn{2}{|c|}{ Von Mises stress of CFL (MPa) } & 22.8 & 24.1 & 24.8 & 25.2 & 45.1 & 47.6 & 48.9 & 49.8 \\
\hline \multicolumn{2}{|c|}{ 1st principal strain of CFL $(m \varepsilon)$} & 87 & 92 & 95 & 97 & 173 & 183 & 188 & 191 \\
\hline \multirow{2}{*}{$\begin{array}{l}\text { Contact stress of } \\
\text { tibiotalar joint }(\mathrm{KPa})\end{array}$} & Tibio cartilage & 0 & 0 & 0 & 0 & 112 & 212 & 249 & 164 \\
\hline & Talar cartilage & 0 & 0 & 0 & 0 & 134 & 267 & 271 & 186 \\
\hline \multirow{2}{*}{$\begin{array}{l}\text { Contact stress of } \\
\text { subtalar joint }(\mathrm{KPa})\end{array}$} & Talar cartilage & 0 & 0 & 0 & 0 & 0 & 0 & 6.2 & 19.9 \\
\hline & Calcaneus cartilage & 0 & 0 & 0 & 0 & 0 & 0 & 6.6 & 20.5 \\
\hline
\end{tabular}

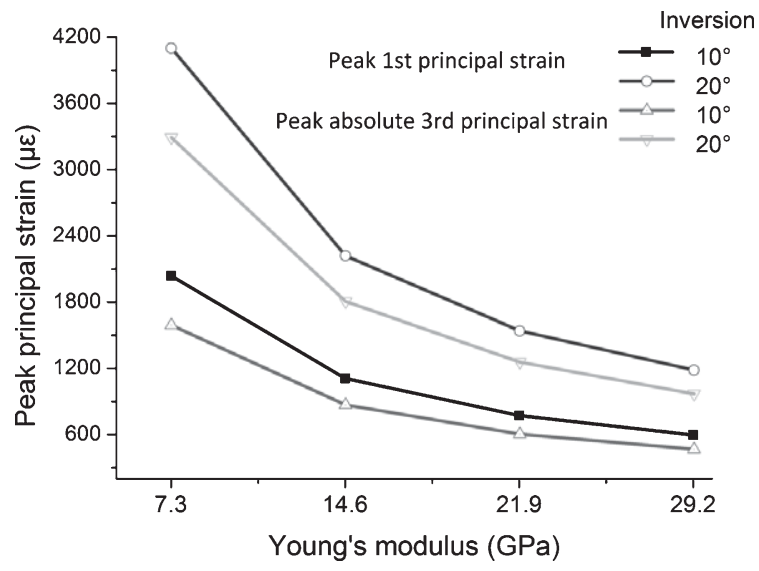

Fig. 3. Changes of the peak 1st and absolute 3rd principal strain with increasing of bone Young's modulus.

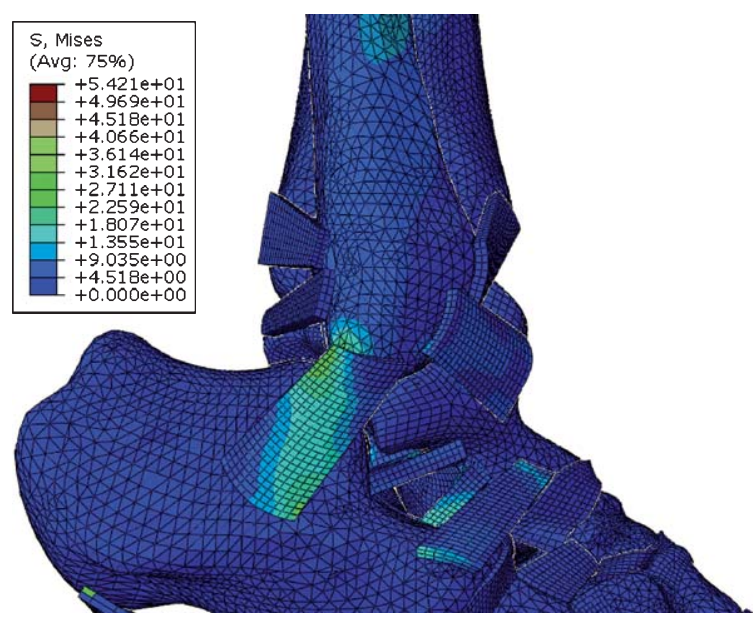

Fig. 4. Distribution of von Mises stress around ankle while $20^{\circ}$ inversion $\left(\mathrm{E}_{\mathrm{Bone}}=29.2 \mathrm{GPa}\right)$. and experimental measurement. While ankle inversion of $20^{\circ}$, the contact stress of the tibiotalar joint increased with increasing of bone Young's modulus. When the Young's modulus was assigned as $29.2 \mathrm{GPa}$, the contact stress of the tibiotalar joint decreased greatly.

\subsection{Experimental measurement}

The strains measured with two gauges were listed as Table 3. Compared to the posterior gauge, the anterior one captured larger strain mean value. However, paired $t$-test found no significant difference between two gauges. Compared to the ankle inversion of $10^{\circ}$, inversion of $20^{\circ}$ produced significant larger strain in both gauges.

\section{Discussion}

Many FE models have been constructed based on $\mathrm{CT}$ or magnetic resonance imaging (MRI). Radiation damage is universally acknowledged as the main disadvantage for CT scan. The abuse of CT-scan on healthy subject even disobeys the ethical standards in some countries. MRI provides fewer details of bony structures compared to CT scan. In this study, CT scan was applied on a cadaveric sample to provide detailed boney features and also to avoid radiation damage for healthy subject. Another consideration is that both computer simulation and experiment used the dead samples. This could avoid the deviations associated with differences between living and dead organisms.

The FEA computation is certainly affected by the material parameters of involved tissues [4]. In 


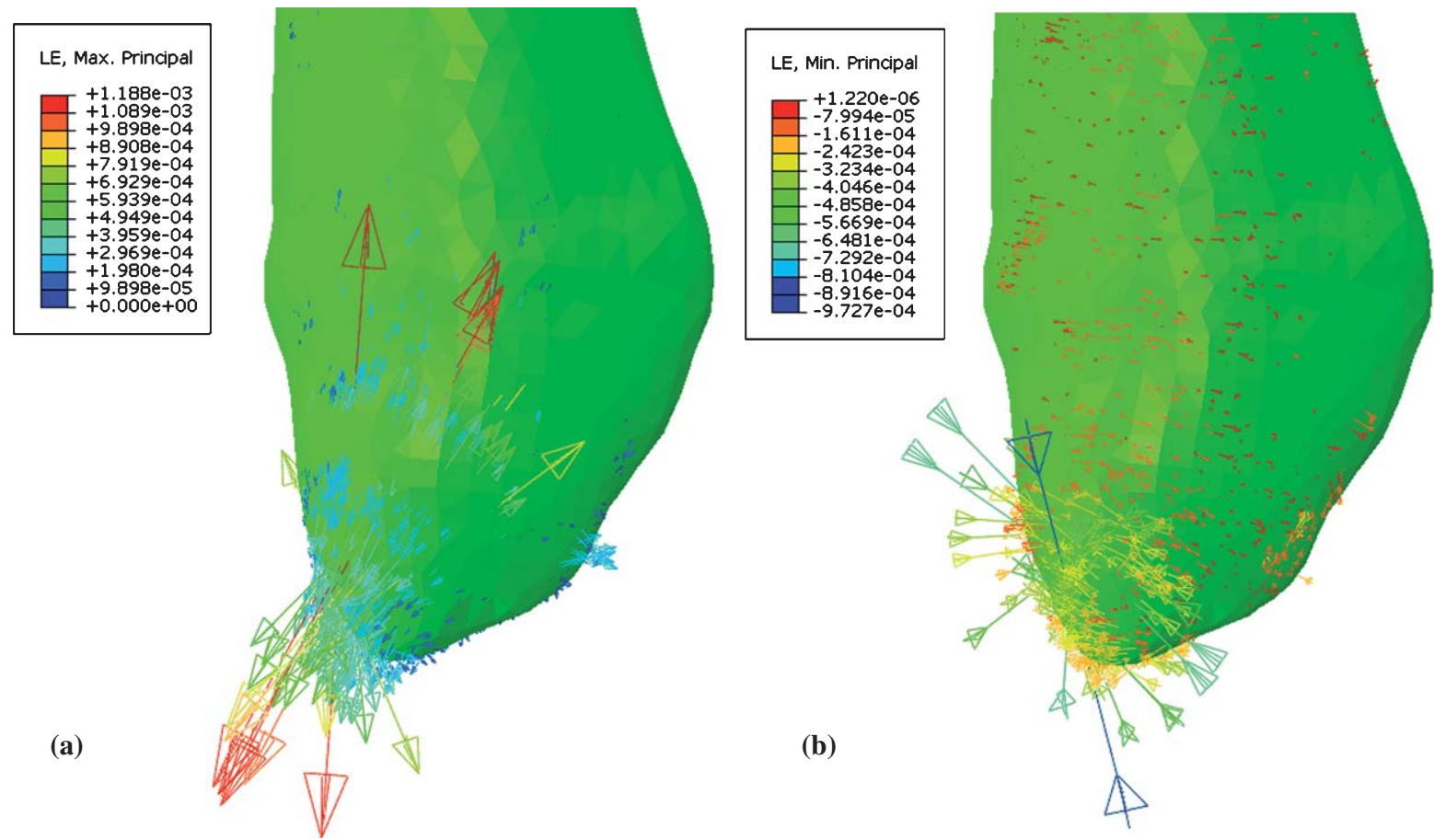

Fig. 5. Distribution vectorgrams of (a) 1 st and (b) 3rd principal strain in the distal fibula.

Table 3

Measurements of two strain gauges in the in-vitro experiment $(\mu \varepsilon)$

\begin{tabular}{lll}
\hline Ankle Inversion & Posterior & Anterior \\
\hline $10^{\circ}$ & $134 \pm 87$ & $180 \pm 89$ \\
$20^{\circ}$ & $275 \pm 188$ & $304 \pm 192$
\end{tabular}

foot-ankle biomechanics, the cortical and trabecular bones were customarily treated as being homogeneous. Factually, they certainly differ in the mechanical property and structure. A review of the bone properties found that more recent experiment measured larger Young's moduli of bones. The pristine experiment usually carried out bulk testing on the entire bone or polished standard sample, which made the measured Young's modulus more comprehensive apparent property of the specimen [27]. New technologies have been used to measure the bone properties in the micro- and nano-structural level for recent years [11]. These measurements considered less influences of the structure and usually got larger Young's moduli of bones than traditional rough methods.

The marrow cavity has not been distinguished from the intact bone in previous FEA studies of foot and ankle [1-9]. In this predicament, it is understandable to assign the apparent Young's modulus of bone. The present study improved the FE model with discrimination of marrow cavity and substantia ossea. The Young's modulus must be increased in the substantia ossea to compensate the stress invalid of the cavity. Therefore, a higher Young's modulus is advised for the FE modeling in the present study.

Stress parameters have been more focused in biomechanics, because larger stress normally means the greater risk in acute disorganization or long-term pathological changes. The stress computation is less determined by the Young's moduli of hard tissues [4], but has more close relation with its geometric structure. However, strain is always directly measured to take the place of stress in experiments. These two parameters, stress and strain are dependent on each other and also different. In FEA study, the strain computation is more determined by the assignment of Young's moduli of involved tissues. If the strain of bone surface was the only concern, the Young's modulus should be assigned greatly as far as possible, because the modulus is larger in the surface cortical bone. If homogeneous property was assumed, the stress distribution output would be less changed by the Young's modulus.

FEA has always been questioned for what extent it can represent the simulated real world. It is necessary 
to validate the model before a FEA report is represented [9]. Two approaches to validate a FE model are experimental validation and model-model validation respectively. The experiments used to validate FE model include in-vivo experiment [2], in-vitro experiment [3, 6,9], or both of them [1]. If an experiment is unavailable for certain study, instead other validated models can also be used as the standard [5].

The results showed great individual variation. The surface strain of the experimental measurement was smaller than the corresponding 1st principal strain peak in FEA. Though they are in the same order of magnitude, the differences are still considerable. Theoretically, the 1st principal strain peak is only in one point, and is highly larger than the strains in the peripheral zone. While the experimental strain measures the mean strain in certain direction and region, which depended on the gauge position and size. The 1st principal strain is the maximum strain of one point in all directions. Its certain direction is hard to be predicted before computation. Though the directions were similar between the strain gauges and the computed 1st principal strain, a tiny angular variation was unavoidable in the experiment. This deviation may greatly influent the experimental strain value. Therefore, the strain difference between in-vitro experiment and FEA is understandable in the present study.

There were some limitations of this study. The function of muscles was not considered in both the computational simulation and in-vitro experiment. Although this would not influent the conclusion, this must be explained when these methods were applied to deal with other problems. We advise a higher Young's modulus $(29.2 \mathrm{GPa})$ used in this study to provide a satisfactory stress and strain outputs. However, this was still not the perfectly factual condition. In the future study, more ideal model should be constructed to represent the nonlinearity, anisotropy and inhomogeneity, as the same time to provide reasonable outputs of the interested parameters.

\section{Conclusion}

The Young's modulus of bone is always assigned with $7.3 \mathrm{GPa}$ in Foot and ankle biomechanics. The assignation of this variable has seldom influence on the stress outputs in the computations. Using the computational simulation and in-vitro experiment, this study advised 4 times of the traditional Young's modulus to get the satisfactory surface strain. The in-vitro experiment is a convenient means to get the real measurement of the surface strain, but more valuable information must be provided with FEA. The 29.2 GPa was in the scope of measured modulus of bones and could be accepted by the researchers.

\section{Acknowledgments}

This work was funded by the Opening Project of Shanghai Key Laboratory of Orthopaedic Implants (KFKT2013002), National Science Foundation of China (NSFC 11302154/11272273/1120101001), Fundamental Research Funds for the Central Universities and National Science \& Technology Pillar Program of China (2012BA122B02).

\section{Conflict of interest}

None.

\section{References}

[1] H.Y. Cheng, C.L. Lin, H.W. Wang and S.W. Chou, Finite element analysis of plantar fascia under stretch-the relative contribution of windlass mechanism and Achilles tendon force, J Biomech 41 (2008), 1937-1944.

[2] J.T. Cheung and M. Zhang, A 3-dimensional finite element model of the human foot and ankle for insole design, Arch Phys Med Rehabil 86 (2005), 353-358.

[3] J.T. Cheung, M. Zhang and K.N. An, Effect of Achilles tendon loading on plantar fascia tension in the standing foot, Clin Biomech (Bristol, Avon) 2 (2006), 194-203.

[4] J.T. Cheung, M. Zhang, A.K. Leung and Y.B. Fan, Threedimensional finite element analysis of the foot during standing - a material sensitivity study, J Biomech 38 (2005), 1045-1054.

[5] J.M. García-Aznar, J. Bayod, A. Rosas, R. Larrainzar, R. García-Bógalo, M. Doblaré and L.F. Llanos, Load transfer mechanism for different metatarsal geometries: A finite element study, J Biomech Eng 131 (2009), 021011.

[6] W.X. Niu, T.T. Tang, M. Zhang, C.H. Jiang and Y.B. Fan, An in-vitro and finite element study of load redistribution in the midfoot. Sci China Life Sci (2014), in press

[7] A. Forstiero, E.L. Carniel and A.N. Natali, Biomechanical behaviour of ankle ligaments: Constitutive formulation and numerical modeling, Comput Methods Biomech Biomed Engin 17 (2014), 395-404.

[8] M. Ni, X.H. Weng, J. Mei and W.X. Niu, Primary stability of absorbable screw fixation for intra-articular calcaneal fractures: A finite element analysis, J Med Biol Eng (2014), Epub ahead of print, Doi: 10.5405/jmbe. 1624 
[9] J. Liang, Y. Yang, G. Yu, W. Niu and Y. Wang, Deformation and stress distribution of the human foot after plantar ligaments release: A cadaveric study and finite element analysis, Sci China Life Sci 54 (2011), 267-271.

[10] S. Sivarasu and L. Mathew, Finite-element-based design optimization of a novel flexion knee used in total knee arthroplasty, Appl Bionics Biomech 5 (2008), 77-87.

[11] Y.D. Gu, J.S. Li, M.J. Lake, Y.J. Zeng, X.J. Ren and Z.Y. Li, Image-based midsole insert design and the material effects on heel plantar pressure distribution during simulated walking loads, Comput Methods Biomech Biomed Engin 14 (2011), 747-753.

[12] S. Nakamura, R.D. Crowninshield and R.R. Cooper, An analysis of soft tissue loading in the foot-a preliminary report, Bull Prosthet Res 10-35 (1981), 27-34.

[13] D.T. Reilly and A.H. Burstein, The mechanical properties of cortical bone, J Bone Joint Surg Am 56 (1974), 1011-1022.

[14] P.J. Thurner, Atomic force microscopy and indentation force measurement of bone, Wiley Interdiscip Rev Nanomed Nanobiotechnol 1 (2009), 624-649.

[15] W. Niu, Z. Chu, J. Yao, M. Zhang, Y. Fan and Q. Zhao, Effects of laterality, ankle inversion and stabilizers on the plantar pressure distribution during unipedal standing, J Mech Med Biol 12 (2012), 1250055 (15 pages)

[16] W.X. Niu, J. Yao, Z.W. Chu, C.H. Jiang, M. Zhang and Y.B. Fan, Effects of laterality, ankle inversion and stabilizers on the plantar pressure distribution during unipedal standing, $J$ Med Biol Eng (2014), Epub ahead of print, Doi: 10.5405/jmbe. 1675

[17] P.G. Pavan, C. Stecco, S. Darwish, A.N. Natali and R. de Caro, Investigation of the mechanical properties of the plantar aponeurosis, Surg Radiol Anat 33 (2011), 905-911.

[18] C. Mkandawire, W.R. Ledoux, B.J. Sangeorzan and R.P. Ching, Foot and ankle ligament morphometry, J Rehabil Res Dev 42 (2005), 809-820.
[19] S. Siegler, J. Block and C.D. Scheck, The mechanical characteristics of the collateral ligaments of the human ankle joint, Foot Ankle 8 (1988), 234-242.

[20] K.A. Athanasiou, J.G. Fleischli, J. Bosma, T.J. Laughlin, C.F Zhu, C.M. Agrawal and L.A. Lavery, Effects of diabetes mellitus on the biomechanical properties of human ankle cartilage, Clin Orthop Relat Res 368 (1999), 182-189.

[21] K.A. Athanasiou, G.G. Niederauer and R.C. Schenck Jr, Biomechanical topography of human ankle cartilage, Ann Biomed Eng 23 (1995), 697-704.

[22] G.T. Liu, L.A. Lavery, R.C. Schenck Jr, D.R. Lanctot, C.F Zhu and K.A. Athanasiou, Human articular cartilage biomechanics of the second metatarsal intermediate cuneiform joint, J Foot Ankle Surg 36 (1997), 367-374.

[23] K.A. Athanasiou, G.T. Liu, L.A. Lavery, D.R. Lanctot and R.C. Schenck Jr, Biomechanical topography of human articular cartilage in the first metatarsophalangeal joint, Clin Orthop Relat Res 348 (1998), 269-281.

[24] D. Wright and D. Rennels, A study of the elastic properties of plantar fascia, J Bone Joint Surg Am 46 (1964), 482-492.

[25] J.R. Funk, G.W. Hall, J.R. Crandall and W.D. Pilkey, Linear and quasi-linear viscoelastic characterization of ankle ligaments, J Biomech Eng 122 (2000), 15-22.

[26] S.P. Nair, S. Gibbs, G. Arnold, R. Abboud and W. Wang, A method to calculate the centre of the ankle joint: A comparison with the Vicon Plug-in-Gait model, Clin Biomech (Bristol, Avon) 25 (2010), 582-587.

[27] F. EI Masri, E. Sapin de Brosses, K. Rhissassi, W. Skalli and D. Mitton, Apparent Young's modulus of vertebral corticocancellour bone specimens, Comput Methods Biomech Biomed Engin 15 (2012), 23-28. 

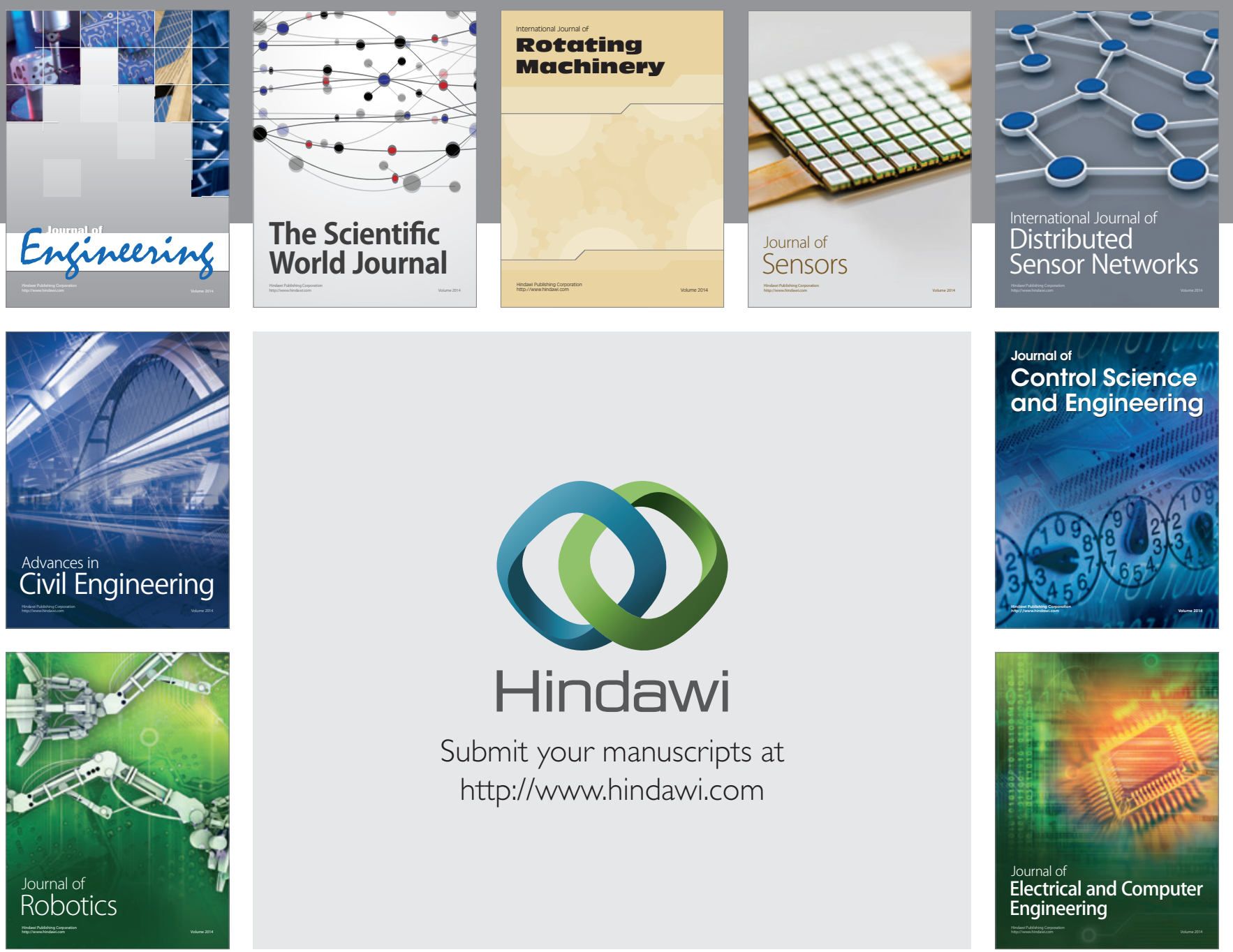

Submit your manuscripts at

http://www.hindawi.com
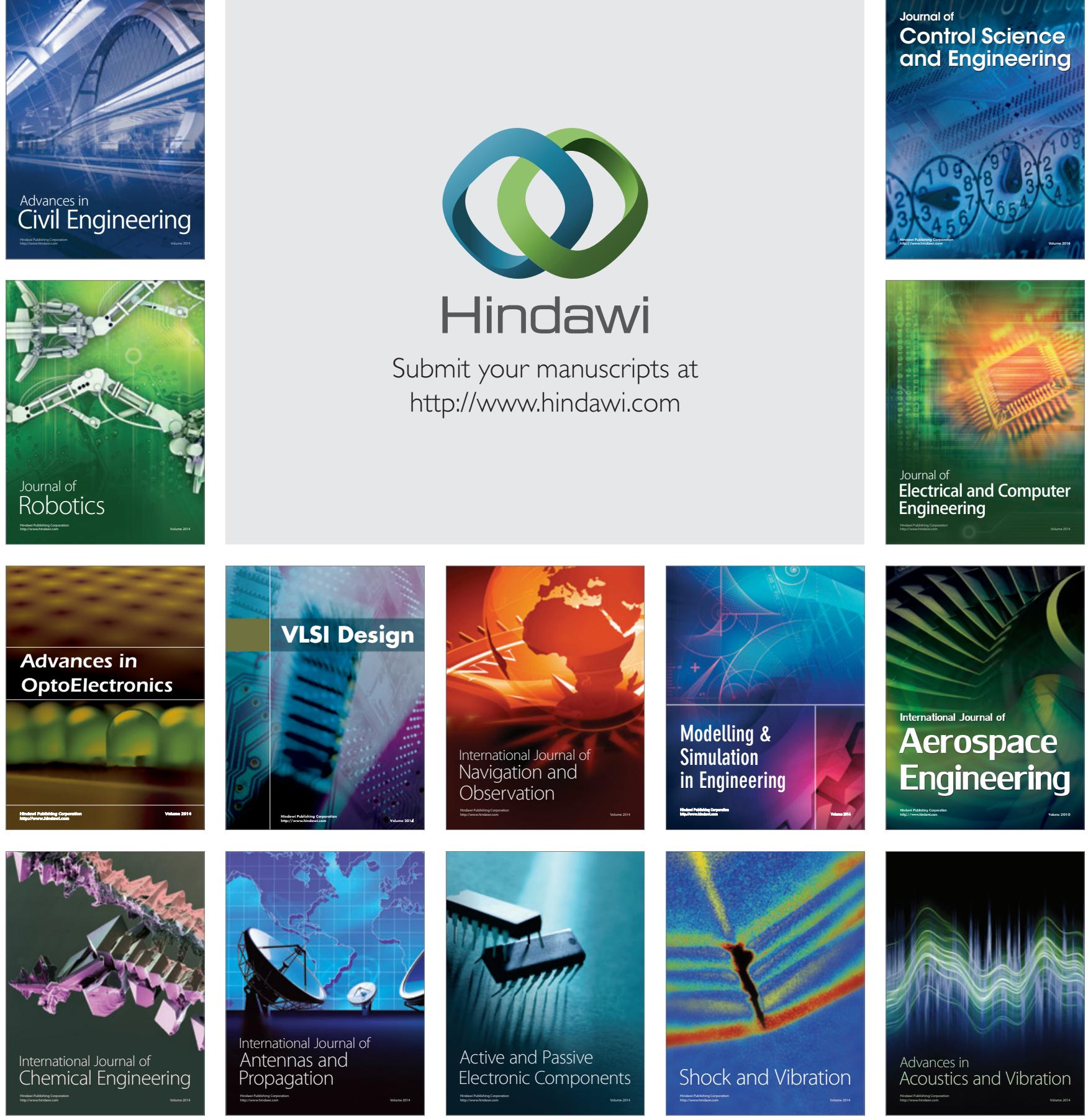